\title{
Evaluation of Cavitation Erosion Behavior of Commercial Steel Grades Used in the Design of Fluid Machinery
}

\author{
I. TZANAKIS, L. BOLZONI, D.G. ESKIN, and M. HADFIELD
}

The erosion response under cavitation of different steel grades was assessed by studying the erosion rate, the volume removal, the roughness evolution, and the accumulated strain energy. A $20 \mathrm{kHz}$ ultrasonic transducer with a probe diameter of $5 \mathrm{~mm}$ and peak-to-peak amplitude of $50 \mu \mathrm{m}$ was deployed in distilled water to induce damage on the surface of commercial chromium and carbon steel samples. After a relatively short incubation period, cavitation induced the formation of pits, cracks, and craters whose features strongly depended on the hardness and composition of the tested steel. AISI 52100 chromium steel showed the best performance and is, therefore, a promising design candidate for replacing the existing fluid machinery materials that operate within potential cavitating environments.

DOI: $10.1007 / \mathrm{s} 11661-017-4004-2$

(C) The Author(s) 2017. This article is published with open access at Springerlink.com

\section{INTRODUCTION}

Cavitation is the repeated formation and violent collapse of bubbles containing vapor and/or gas in a liquid caused by periodic tensile stresses imposed onto the liquid phase. Cavitation can be induced by high-frequency vibrations (acoustic cavitation) or by sudden pressure variation in the flow (hydrodynamic cavitation). In the latter, at a critical pressure, cavitation can be initiated by the localized phase change (partial liquefaction), from vapor to liquid, e.g., by a high-velocity working gas as in scroll expander systems. ${ }^{[1,2]}$ When cavitation bubbles implode close to a surface, powerful micro-jets of velocity in the range of 300 to $1000 \mathrm{~m} / \mathrm{s}$ with hydrodynamic impact pressures of more than $1 \mathrm{GPa}$ are produced. ${ }^{[3,4]}$ These micro-jets in combination with the pressure waves emitted during the implosion of cavitation bubbles promote the formation of incubation pits of various sizes and shapes across solid surfaces. ${ }^{[5-7]}$ The cyclic nature of the surface stress leads to the coalescence of the incubation pits and consequently to the typical cavitation erosion damage (i.e., formation of deep craters and cracks). ${ }^{[5,8]}$

Cavitation erosion is the major failure mechanism of many fluid machinery components, being responsible

I. TZANAKIS is with Brunel Centre for Advanced Solidification Technology (BCAST), Brunel University London, Uxbridge, Middlesex, UB8 3PH, UK, and also with Oxford Brookes University, Faculty of Technology, Design and Environment, Oxford, OX33 1HX, UK. Contact e-mail: itzanakis@brookes.ac.uk L. BOLZONI is with the Faculty of Science and Engineering, Waikato University, Hamilton 3240, New Zealand. D.G. ESKIN is with Brunel Centre for Advanced Solidification Technology (BCAST), Brunel University London, and also with Tomsk State University, Tomsk, 634050 Russia. M. HADFIELD is with the Department of the Design and Engineering, Bournemouth University, Poole, BH12 5BB, UK.

Manuscript submitted June 24, 2016.

Article published online March 6, 2017 for high maintenance costs. Therefore, cavitation erosion rate and resistance are important parameters required for designing hydraulic parts and estimating their in-service performance. This is especially true when cavitation cannot be avoided due to design limitations, such as within scroll expander systems where the working fluid transfers energy to the rotor. Thus, a proper selection of materials for critical components with high cavitation erosion resistance is a necessity. Moreover, the damaged surface of eroded components may induce perturbations of the fluid flow; and the overall pressure ratio as well as the operating efficiency can be significantly decreased. ${ }^{[9,10]}$

The most common method to simulate short-period (incubation pits) and long-period (craters formation) cavitation erosion at the laboratory scale is by using high-frequency sound waves generated by an ultrasonic device. Ultrasonic vibrations are introduced into the liquid by a sonotrode (ultrasonic horn). The sonotrode tip is immersed in a given liquid to transmit ultrasonic vibrations that can produce a phenomenon called acoustic cavitation (identical in produced effects to hydrodynamic cavitation). Several parameters are known to have a major influence on the erosive potential of the cavitation bubbles: (i) the viscous and surface tension forces of the liquid environment, (ii) the distance of the bubble to the wall interface, (iii) the maximum size of the bubble prior to collapse, and (iv) the adverse pressure gradient to which the bubble is subjected and which causes its collapse. ${ }^{[11]}$ It has been reported that the cavitation erosion resistance of materials, even if it can be regarded as an independent mechanical property of the material itself ${ }^{[12]}$ depends on mechanical properties and characteristics such as strain energy, ${ }^{[13]}$ ultimate strength, ${ }^{[14]}$ hardness,${ }^{[15]}$ roughness ${ }^{[16]}$ as well as the strain hardening ability of the material. ${ }^{[17]}$ Thus, extensive research has been carried out elsewher ${ }^{[18-23]}$ in order to find effective correlations between the 
cavitation erosion rate and the physical properties of the tested fluids as well as the cavitation erosion rate and the mechanical properties of the tested materials.

In particular, Hattori et al. ${ }^{[24,25]}$ reviewed extensive reference data on the cavitation erosion resistance of various steel grades and showed that carbon concentration in steels is a critical parameter in cavitation erosion. Hattori et al. showed that the mean depth erosion rate (MDER) of steel grades, especially for those with carbon content lower than $0.10 \mathrm{wt}$ pct, is approximately twice as that of carbon steels with carbon content $0.15 \mathrm{wt}$ pct with similar hardness, ranging from 20 to $40 \mu \mathrm{m} / \mathrm{h}$ during the first 5 hours of test. Haosheng et al. ${ }^{[26]}$ identified the typical stages of cavitation erosion for a common chromium steel 40Cr (equivalent to AISI 4140) according to the mass loss curves and found that after 5 hours of exposure to acoustic cavitation, the MDER was in the range of $5.5 \pm 0.5 \mu \mathrm{m} / \mathrm{h}$. Dojicinovic et al..$^{[27]}$ determined the MDER of unalloyed austempered ductile iron to be around $4.5 \pm 0.5 \mu \mathrm{m} / \mathrm{h}$ after 4 hours of testing. Steller, ${ }^{[28]}$ using vibratory equipment, reported the MDER of two different steel types: a medium carbon steel C45 (equivalent to AISI 1045) and a stainless steel $1 \mathrm{H} 18 \mathrm{~N} 9 \mathrm{~T}$ where he found that after 8 hours of exposure to cavitation, the MDER was approximately $17 \pm 1 \mu \mathrm{m} /$ $\mathrm{h}$ for the carbon steel and $20 \pm 1 \mu \mathrm{m} / \mathrm{h}$ for the stainless steel. Chiu et al. ${ }^{[29]}$ investigated the cavitation behavior of AISI 316L stainless steel finding that the MDER after 5 hours of test is about $3 \pm 0.5 \mu \mathrm{m} / \mathrm{h}$. Finally, Kwok et al. ${ }^{[30]}$ evaluated the MDER of a mild steel AISI 1050 exposed to cavitation in $3.5 \% \mathrm{NaCl}$ solution for a prolonged period of time. The MDER was estimated as $12 \pm 1 \mu \mathrm{m} / \mathrm{h}$.

In spite of many years of fundamental research on this topic, the quantification of the erosive potential of collapsing bubbles and the rationalization of the cavitation erosion process remains a challenge. This is especially the case with real engineering systems operating within cavitating environments. The starting point of this work is the analysis of a scroll expander system, manufactured from hardening steel (international equivalent to AISI W1 or ASTM A686), which had been integrated into a micro-combined heat and power ( $\mu$-CHP) unit. It was found that the scroll experienced significant cavitation erosion near to the inlet after 1000 hours of continuous service (evidence of cavitation pitting was found after the first 300 hours of continuous service $^{[1,10,31]}$ ). The performance of the $\mathrm{W} 1$ steel plate of the scroll expander was then compared-using a designed experiment with acoustically induced cavitation-to that of other commercial steel grades, i.e., possible candidates for replacing the currently used grade. The aim of this study is to assess and discuss the durability performance of the different steel grades after the incubation period (that was covered in previous research work $\left.^{[31]}\right)$. The primary focus of the current investigation is therefore the progression of the cavitation erosion as well as the design assessment of the cavitation erosion performance of the studied steel grades after prolonged periods of time by evaluating their morphology evolution, volume loss, roughness profile, accumulated strain energy and hardness. Results can be used for the selection of a steel material for an adequate performance against cavitation erosion based on the erosion resistance ranking of the tested materials.

\section{EXPERIMENTAL PROCEDURE}

Accelerated erosion laboratory tests using an ultrasonic transducer were performed, and a comparative study among the tested materials at different erosion stages was conducted. The laboratory experimental studies aim at revealing the cavitation erosion resistance of the tested materials within relatively short periods of time, whereas under the real operation conditions the cavitation erosion is expected to occur only when the exposure time is significantly longer (e.g., the cavitation erosion is clearly observed within scroll systems after 1000 hours of continuous service).

\section{A. Surface Examination}

This commenced with a detailed surface examination of the steel plate (AISI W1/ASTM A686) used within the scroll expander system of a $\mu$-CHP (combined heat and power) appliance unit exposed to a service period of 1000 hours. Cavitation damage across the surface of the steel plate was identified by scanning electron microscopy (SEM) and quantitatively analyzed with the use of a high-precision $3 \mathrm{D}$ optical interferometer microscope (ZYGO).

\section{B. Studied Materials}

Steel grades, which are usually inexpensive and exhibit considerable cavitation erosion resistance, are widely used in fluid machinery design. In this study, three standard commercial steel grades with international equivalents AISI 52100, AISI 1020, AISI 1085 and with a lower cost than that of the actual steel AISI W1 plate currently used in a scroll expander were selected. These materials were chosen as they cover a wide range of steel grades and mechanical properties. According to the structure analysis preformed in Reference 31, the steel plate of the scroll (AISI W1) is classified as a quench-hardened tool steel with martensitic structure, the AISI 52100 grade is classified as chromium steel with martensitic structure and predominantly spheroidal carbides, AISI 1020 is a low-carbon steel grade with tempered martensitic structure and small amounts of ferrite and retained austenite and AISI 1085 is a high-carbon steel grade with small amounts of bainitic and ferritic constituents in a matrix of tempered martensite.

The heat treatment conditions were chosen to achieve a range of mechanical properties rather than to match the practically used heat treatment regimes for specific applications. The chemical composition and relevant mechanical properties of these materials are shown in Tables I and II, respectively, as measured using Optical Emission Spectrometer (OES) and appropriate laboratory equipment such as a tensile testing machine (Testometric) and hardness test. 
Table I. Chemical Analysis of the Steel Samples Used for Cavitation Tests

\begin{tabular}{lccccccccc}
\hline & \multicolumn{7}{c}{ Chemical Composition Weight Percent } \\
\cline { 2 - 9 } Steel Grades & $\mathrm{C}$ & $\mathrm{Si}$ & $\mathrm{S}$ & $\mathrm{Mn}$ & $\mathrm{Cr}$ & $\mathrm{P}$ & $\mathrm{Al}$ & $\mathrm{Ni}$ & $\mathrm{Cu}$ \\
\hline AISI W1 & 0.96 & 0.27 & 0.003 & 0.46 & 0.18 & 0.018 & - & 0.2 & 0.1 \\
AISI 52100 & 1 & 0.23 & 0.004 & 0.31 & 1.53 & 0.012 & 0.008 & 0.011 & 0.13 \\
AISI 1020 & 0.24 & 0.16 & 0.024 & 0.54 & 0.03 & 0.014 & - & 0.01 & - \\
AISI 1085 & 0.83 & 0.23 & 0.005 & 0.57 & 0.05 & 0.008 & 0.005 & 0.06 & 0.1 \\
\hline
\end{tabular}

Table II. Typical Mechanical Properties of the Steel Samples Used for Cavitation Tests

\begin{tabular}{|c|c|c|c|c|}
\hline \multirow[b]{2}{*}{ Material Properties } & \multicolumn{4}{|c|}{ Steel Grades } \\
\hline & AISI W1 & AISI 52100 & AISI 1020 & AISI 1085 \\
\hline Tensile Strength (MPa) & $1650 \pm 20$ & $2240 \pm 10$ & $365 \pm 10$ & $735 \pm 20$ \\
\hline Yield Strength (MPa) & $1500 \pm 20$ & $2030 \pm 10$ & $300 \pm 10$ & $540 \pm 10$ \\
\hline Elongation at fracture $(\%)$ & $4 \pm 0.5$ & $5 \pm 0.5$ & $18 \pm 1$ & $10 \pm 1$ \\
\hline Density $\left(\mathrm{kg} / \mathrm{m}^{3}\right)$ & 7825 & 7835 & 7860 & 7860 \\
\hline Hardness (Vickers) & $540 \pm 10$ & $860 \pm 10$ & $350 \pm 10$ & $865 \pm 10$ \\
\hline
\end{tabular}

The as-quenched martensitic steel specimens had low toughness and high residual stresses. Therefore, hardened carbon steels should be tempered after hardening in order to prevent cracking and increase toughness. Samples of the tested steel grades were supplied by a commercial supplier with the following heat-treated conditions:

- AISI 1095 steel was heated beyond its transition temperature $>1011 \mathrm{~K}\left(738{ }^{\circ} \mathrm{C}\right)$ and then quickly quenched in oil, followed with tempering at $643 \mathrm{~K}$ $\left(370{ }^{\circ} \mathrm{C}\right)$.

- AISI 1020 steel was heated to the range $1153 \mathrm{~K}$ to $1193 \mathrm{~K}\left(880{ }^{\circ} \mathrm{C}\right.$ to $\left.920{ }^{\circ} \mathrm{C}\right)$ in a suitable carburizing atmosphere followed by quenching. It was then tempered at $423 \mathrm{~K}\left(150{ }^{\circ} \mathrm{C}\right)$.

- AISI 52100 steel was vacuum degassed steel heated up to $1103 \mathrm{~K}\left(830^{\circ} \mathrm{C}\right)$, followed by quenching in oil and tempering at $473 \mathrm{~K}\left(200{ }^{\circ} \mathrm{C}\right)$.

- AISI W1 steel was heated to $1048 \mathrm{~K}\left(775{ }^{\circ} \mathrm{C}\right)$ followed by water quenching and tempering at 603 $\mathrm{K}\left(330^{\circ} \mathrm{C}\right)$.

For the cavitation erosion study, the surfaces of steel plates were finely polished by diamond suspension and cleaned with acetone in an ultrasonic bath $(40 \mathrm{kHz})$ for a few seconds. In each case, the surface finishing (arithmetic average $\mathrm{Ra}$ roughness parameter) of the starting materials was lower than $0.05 \mu \mathrm{m}$ as measured using an interferometer microscope.

\section{Cavitation Erosion Test}

Cavitation erosion tests were carried out based upon the set up shown in References 3, 5, and 32. An ultrasonic transducer at a frequency of $20 \mathrm{kHz}$ and a peak-to-peak vibratory amplitude of $50 \mu \mathrm{m}$ was deployed. Vibrations were introduced in the liquid media using a stepped titanium probe with the $5 \mathrm{~mm}$ diameter. Steel samples were mounted on the bottom of

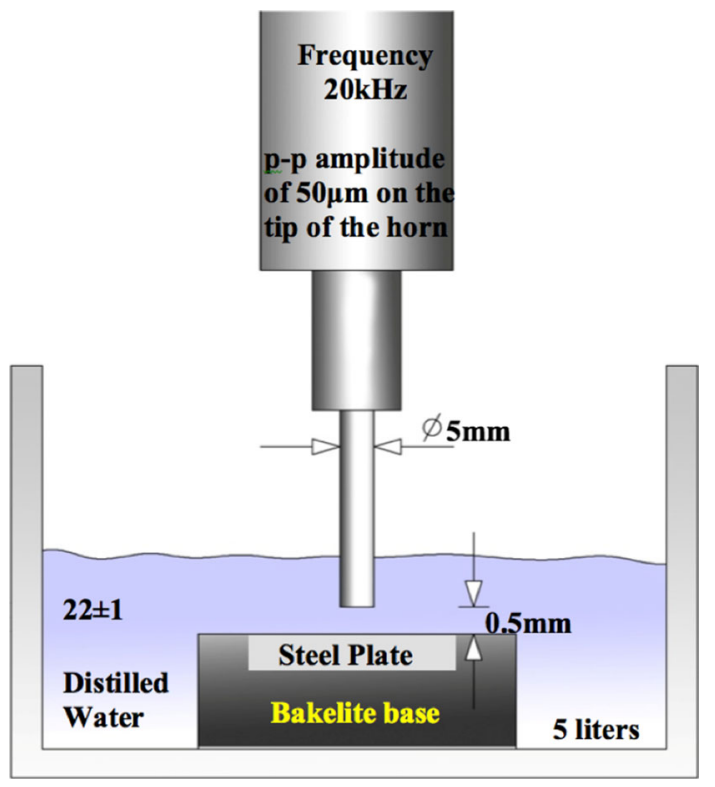

Fig. 1-Schematic overview of the ultrasonic vibratory method used for cavitation tests.

a large transparent tank (dimensions $40 \times 20 \times 15 \mathrm{~cm}^{3}$ ) filled with 5 liters of distilled water. The tip of the titanium horn was submerged to a distance of $0.5 \mathrm{~mm}$ from the top of the steel sample (Figure 1). Cavitation created by the small size probe generated a gradual damage that was useful for studying the mechanisms of cavitation erosion, while the energy released by cavitation did not significantly affect the water temperature that was maintained at $295 \pm 1 \mathrm{~K}\left(22 \pm 1^{\circ} \mathrm{C}\right)$.

Steel samples were exposed to cavitation damage for periods of time ranging from 1 to 8 hours, which is much shorter than in-service exposure times. At the final time step, a local equilibrium between the erosive power of the cavitation field and the response from the studied material occurred, meaning that the steady state period 
for that particular sample was achieved (see Section IV). The steady state or terminal stage is when the rate of weight loss reaches a quasi-constant value (balance of energy accumulated and lost with removed material particles $)^{[32]}$ and a decline tendency is shown. ${ }^{[33]}$ This implies that the candidate material that reaches the stable and the decline stage first exhibits a superior cavitation erosion resistance and excessive damage is restricted. In order to obtain consistent and comparable data, each test was carried out at least 4 times.

\section{Surface Analysis and Characterization}

After the completion of each experiment, specimens were removed from the tank and cleaned. Cavitation damage was analyzed using the same procedure as described in Section II-A. The durability and resistance against cavitation erosion were evaluated by measuring the volume loss and the evolution of the roughness profiles using an advanced high-precision 3D ZYGO interferometer microscope. Volume loss was calculated from the difference between the actual worn surface and the reference plane of the intact region around it. The MDER was determined as the tangent to the cumulative erosion-time curve (expressed in $\mu \mathrm{m} / \mathrm{h}$ ), and the cavitation erosion resistance (CER) was defined as the reciprocal of the mean erosion rate (MDER). MDER and CER were calculated using Eqs. [1] and [2], respectively:

$$
\begin{aligned}
& \text { MDER }=10 \frac{\Delta V}{A \cdot \Delta t}(\mu \mathrm{m} / \mathrm{h}) \\
& \mathrm{CER}=\operatorname{MDER}^{-1}(\mathrm{~h} / \mu \mathrm{m})
\end{aligned}
$$

where $\Delta V$ is the volume loss, $\rho$ is the density of the material, $A$ is the cavitation-affected area of the sample, and $\Delta t$ is the test time.

Another important parameter, which characterizes the energy absorbing capacity of the metals under the repeated indenting loads by the implosion of the cavitation bubbles and is related to the cavitation erosion evolution, is the strain energy. Cavitation erosion damage is considered to be a fatigue phenomenon because material removal occurs after the repeated impacts due to the implosion of bubbles. The accumulated strain energy can be used to identify the point where damage becomes critical for different materials. ${ }^{[34]}$ Both the elastic and the plastic behavior of the material may affect this accumulated energy and, consequently, data can be analyzed on the basis of either purely elastic or elastoplastic behavior. Thus, for example, when brittle materials are considered, the accumulated elastic strain is more appropriate to be used, although when ductile or partially ductile materials are examined (as in this study), then it should be more appropriate to use the total energy strain (plastic and elastic strain energy). However, as material's behavior could rapidly change during the cavitation process, e.g., work hardening and related accumulation of defects and restriction of dislocation movement leading to increment of material brittleness, the authors found it useful to examine the behavior of the tested materials under both of these strain energy conditions for better understanding and fuller characterization of mechanical behavior under repeated loads. Thus, the strain energy was correlated to the volume loss in order to estimate the cavitation damage intensity (Section IV). The accumulated elastic strain energy $\mathrm{E}_{\mathrm{el}}$ and the total strain energy $E_{\text {tot }}$ estimated the rate of the erosion and the resistance of the samples against cavitation and are expressed by Eq. [3] ${ }^{[35]}$ and Eq. [4], ${ }^{[36]}$ respectively.

$$
\begin{gathered}
E_{\mathrm{el}}=V \cdot u=V\left(\sigma_{y} / 2 E\right)(\mathrm{mJ}) \\
E_{\mathrm{tot}}=V \cdot u^{*}=V\left(\sigma_{\mathrm{ts}}+\sigma_{y}\right) \varepsilon / 2(\mathrm{~mJ}),
\end{gathered}
$$

where $V$ is the volume loss, $u$ is the elastic strain energy density per unit volume, $u^{*}$ is the total strain energy density per unit volume, $\sigma_{\text {ts }}$ is the ultimate tensile strength, $\sigma_{\mathrm{y}}$ is the yield strength, $\varepsilon$ is the fracture elongation, and $E$ is the Young modulus of the material. The mean maximum depth of the damage formed at the centre of the samples during the cavitation erosion period provided the physical interpretation of individual damage periods. The profile of the damage was evaluated using optical interferometer microscopy and the crater growth rate among the different stages was estimated using Eq. [5]:

$$
I_{D}=\frac{D_{i}-D_{0}}{D_{i}} \times 100(\mathrm{pct})
$$

where $I_{\mathrm{D}}$ is the mean percentage of increment (percentage change) of the maximum depth of the crater between different time stages $D_{i}$ and $D_{0}$.

Finally, hardness was measured across the eroded regions of the test samples in order to verify the existence of work-hardened subsurface layers affected by cavitation. The indentations were performed using a Vickers microhardness tester with a loading of $10 \mathrm{~N}$. For each time step, a number of 10 measurements were carried out on the exposure surface and the average was taken. The hardness of the eroded materials was correlated with the erosion resistance.

\section{OBSERVATIONS AND MEASUREMENTS}

\section{A. Characterisation of an Eroded Scroll Expander Part}

A detailed surface examination of the AISI W1 steel plates (Figure 2(c)) of the scroll expander system, after its service period of 1000 hours as the main part of an experimental $\mu$-CHP unit, was performed. The scroll expander and its individual components are shown in Figure 2. A full account upon the specific scroll expander technology and its operational conditions can be found in Reference 37. Results showed that cavitation erosion across the steel plate of the scroll expander is one of the main mechanisms, apart the primary tribological mechanisms governing the contact of the two involute spiral scrolls discussed in previous studies, ${ }^{[38,39]}$ which can seriously affect the lifecycle of a scroll system. 


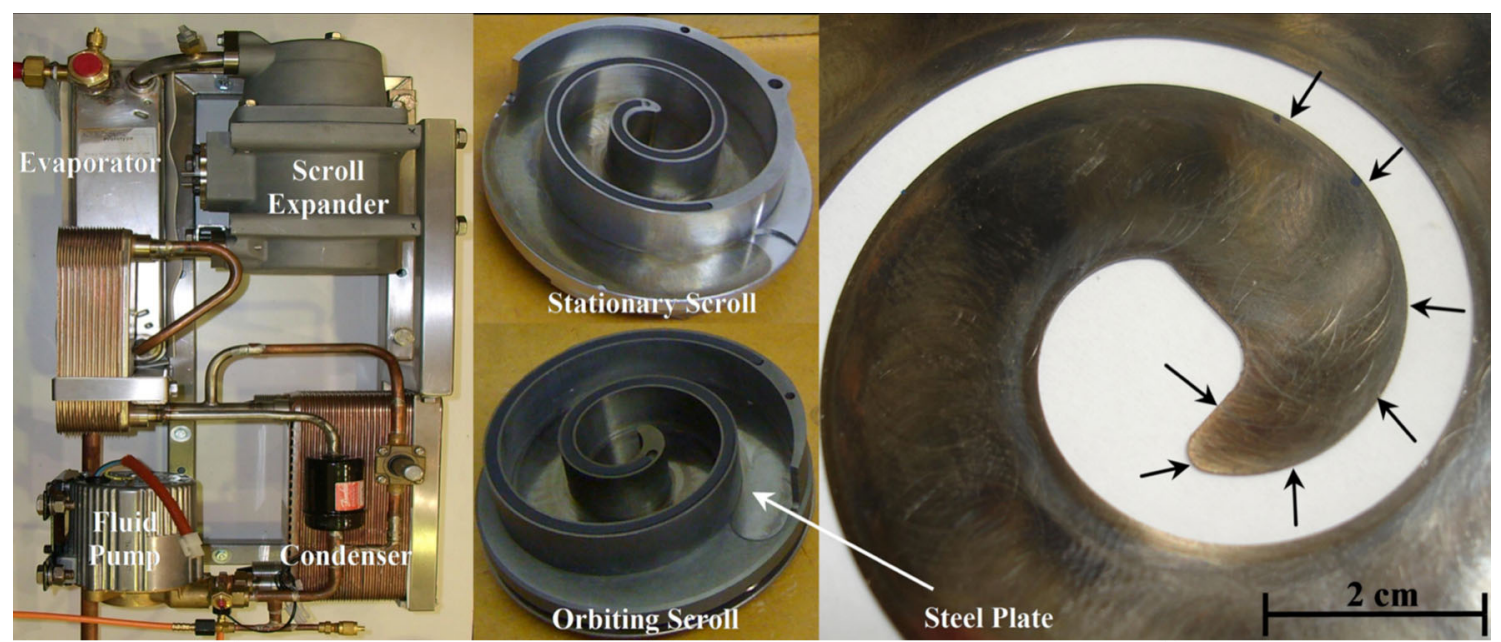

(a)

(b)

(c)

Fig. 2-Photos of the $\mu$-CHP unit studied: $(a)$ assembled system, $(b)$ stationary and orbiting involute scrolls, and $(c)$ cavitation-affected zones on the scroll plate analyzed.

Specifically, many cavitation pit clusters were identified across the steel plate (indicated by black arrows in Figure 2(c) and seen in detail in Figure 3), in the area close to the high-pressure region in the suction port of the scroll. The cavities were mainly accumulated along the edges of the steel plate, creating a continuous layer of cavities of approximately $1.5 \mathrm{~mm}$ wide. In the low-pressure regions of the steel plate, no traces of cavities were found. The mechanism dominating the formation of cavities in these particular high-pressure regions was explained in detail elsewhere. ${ }^{[1,2]}$ The appearance of the eroded steel plate where cavity clusters were formed is shown in Figure 3(a). These clusters consisted of cavitation pits, which accelerated the cavitation process by forming deeper and wider cavities that would be eventually transformed into deep craters. Dular et al. ${ }^{[40]}$ stated that these cavity clusters varied significantly in size and shape compared to individual cavitation pits and they tended to grow much faster. Due to their arbitrary and irregular shape, they became favorable nucleation spots for cavitation bubbles to grow and to implode, leading to the reduction of the resistance of the material in that region. Typical examples of the cavity clusters found in the steel plate are shown Figure 3(b).

From interferometer data (Figure 3(c)), key dimensions (depth and diameter) of the individual pits found across the steel plate were accurately calculated and presented as histograms of Figure 4. A total number of 110 individual pits were analyzed across the steel plate. The frequency distribution shows the probability of these two geometrical parameters among the measured cavitation pits. The average diameter was in the range of 10 to $20 \mu \mathrm{m}$, while the average depth was in the range of 1 to $2 \mu \mathrm{m}$. The probability of cavitation-induced pits being larger than $3 \mu \mathrm{m}$ inside the scroll after that particular time duration was low, but still quite noticeable (about 20 pet possibility). Similarly, the probability for the formation of pits with diameters higher than 30 $\mu \mathrm{m}$ was low. The growth of cavitation clusters was caused largely by linking up of nearby cavitation pits. The process of their enlargement, transforming their shape into deep craters, was dependent on time and history. According to Howell, ${ }^{[41]}$ the gap between the scrolls is typically $1 \mu \mathrm{m}$ across. However, this may be increased by cavitation impacts, and it is known that if it reaches around $8 \mu \mathrm{m}$, the scroll can become inefficient.

\section{B. Simulated Erosion}

The cavitation pits formed in the early stages of cavitation (described in Reference 31) significantly grew by agglomeration of nearby pits, cracks, and grooves. The surface roughness substantially increased, accelerating the erosion rate of the materials. The enlargement of the pits was accompanied by the additional volume loss. The mechanism of pit growth leads to the formation of deep and wide craters penetrating the surface of the sample. These regions are called "cavitation ring areas" because of their typical shape and an example, which is representative for the four steel grades studied, is shown in Figure 5.

Three different areas can be distinguished in the damage profile as shown in Figure 5(c): the centre (crater), the periphery (outer annulus), and the area between those two (reference area). Cavitation erosion is substantially larger, deeper, and wider in the centre compared to the periphery. The lowest damage is found in the area between the centre and the periphery (reference area) where erosion is superficial, being dominated by isolated cavitation pits (Figure 5(b)). A detailed description of this grading of erosion damage can be found elsewhere. ${ }^{[42]}$ The intensity of cavitation is accumulated in the centre of the sample, as clearly indicated by the direction of the acoustic stream in Figure 5(a), (a full account of the cavitation high-speed monitoring process can be found in Reference 18) and also explained by Moussatov et al.$^{[43]}$ by the conicity of the acoustic stream where streamlines tend to focus in a centre point. Therefore, the damage in that particular 

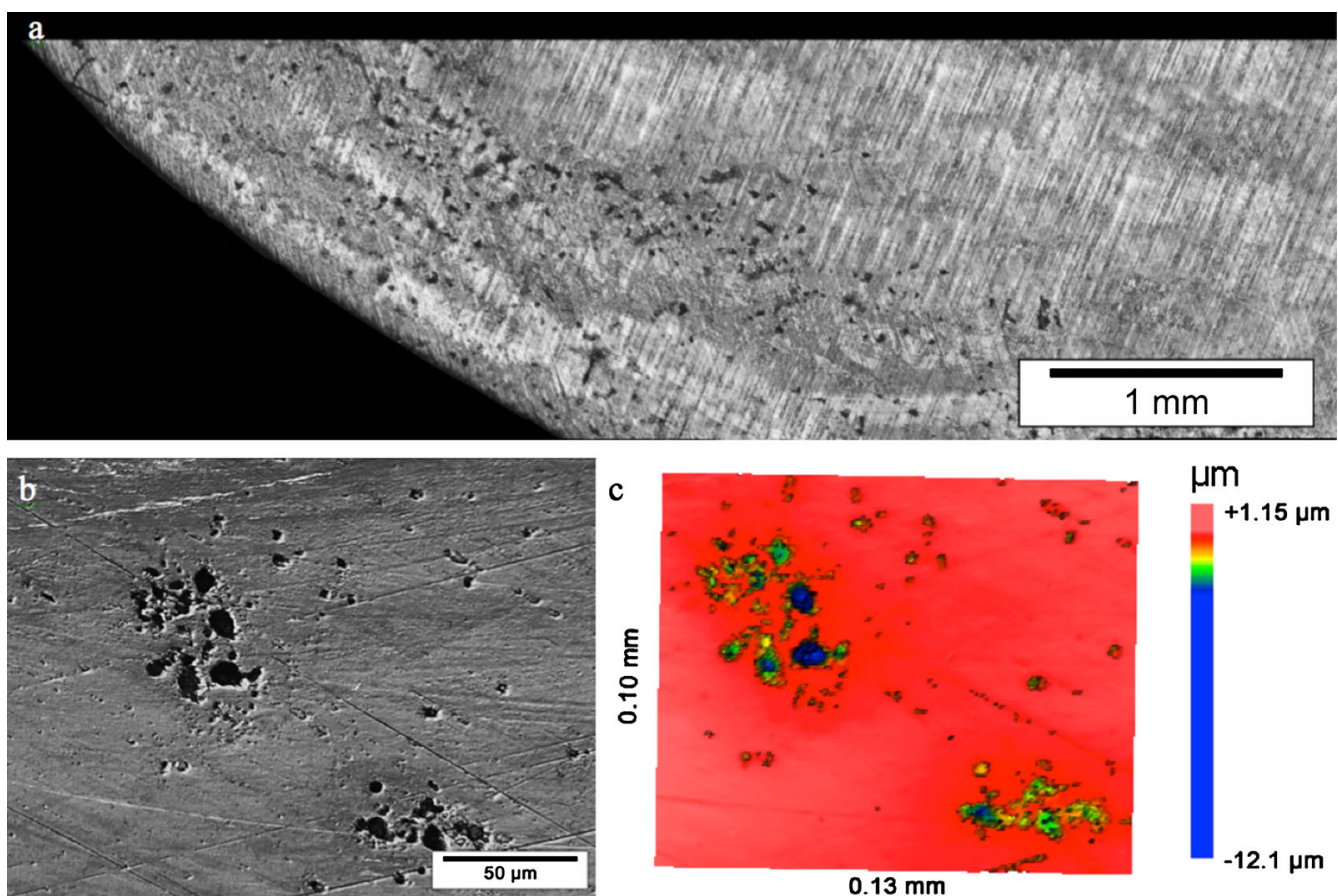

Fig. 3-Details of the cavitation eroded AISI W1 steel plates: (a) cavity clusters found in the high-pressure region of the scroll expander, (b) optical micrograph of the cavity clusters, and $(c)$ ZYGO interferometer analysis of the cavity clusters.

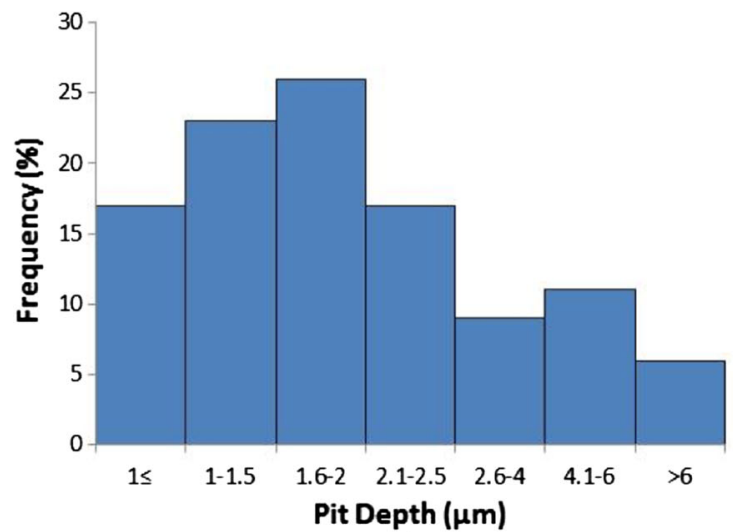

(a)

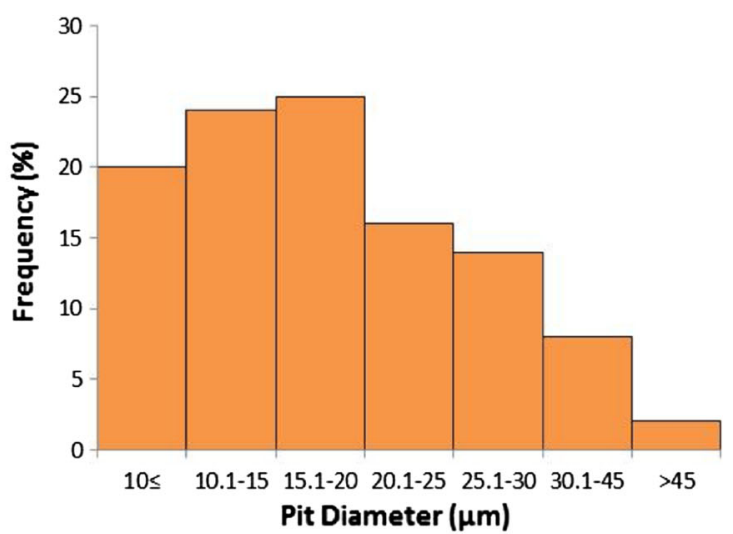

(b)

Fig. 4-Histogram of $(a)$ pit depth and $(b)$ pit diameter distribution in the actual part of the scroll steel plate after its service period of $1000 \mathrm{~h}$. Results are from the analysis of 110 pits.

area occurs continuously. In contrast, in the areas outside the crater, a damage pattern takes time to be formed and is more stochastic. As a result, erosion is randomly distributed without any consistent pattern other than the formation of an outer annulus as explained in Reference 42. Only the central eroded area (the crater) is considered to be representative for the erosion tests and thus is taken into account for comparing different steel grades.

Although the steel samples are characterized by different microstructural features (such as the presence of carbides) and different cavitation erosion resistance, they share similar brittle pattern of surface damage. This is illustrated in Figure 6 where SEM and optical interferometer microscope micrographs are given. The damaged surfaces are characterized by the formation of pits, undulations, fatigue cracks, deep craters, and protruding steps as visible in the SEM images. The erosion features of the final stage are depicted by the interferometric micrographs. At the same time, each steel has some specific manifestations of the common features.

Figure 6(a) shows the initial stage of erosion pits and cracks formation after 1 hour of testing for the AISI W1 steel sample. Erosion mainly originated from pits and cleavage with their adjacent areas plastically deformed. As the cavitation time further increased, the pits coalesced and formed a larger crater. Gradually, 


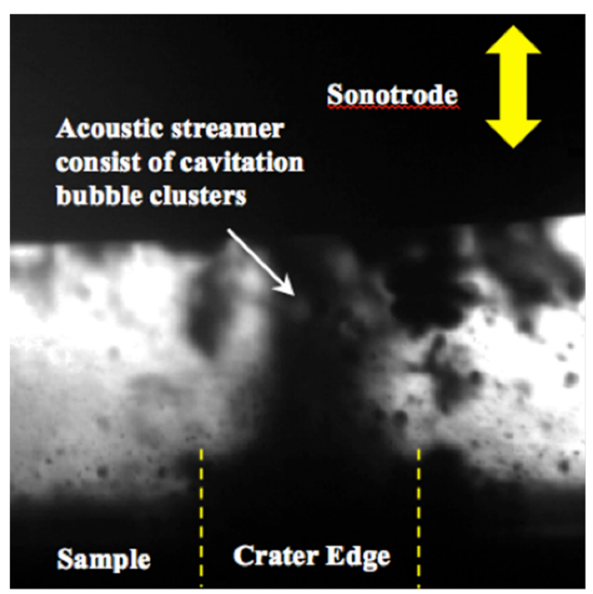

(a)

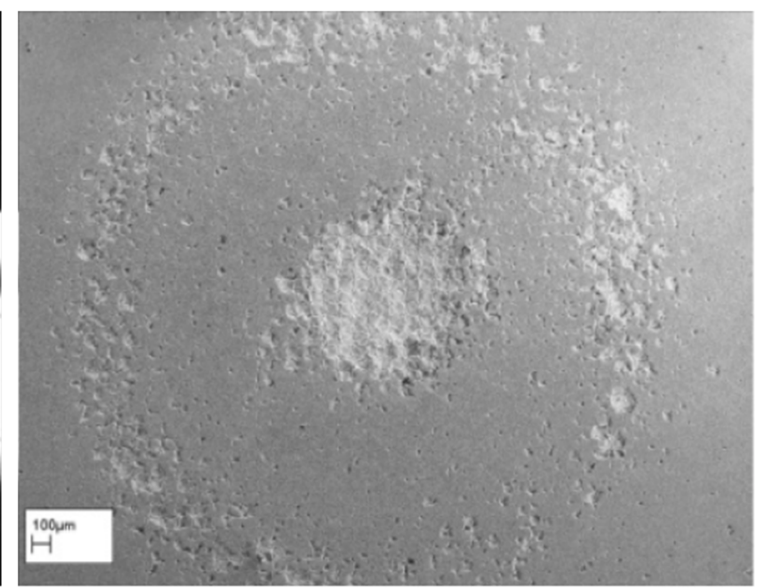

(b)
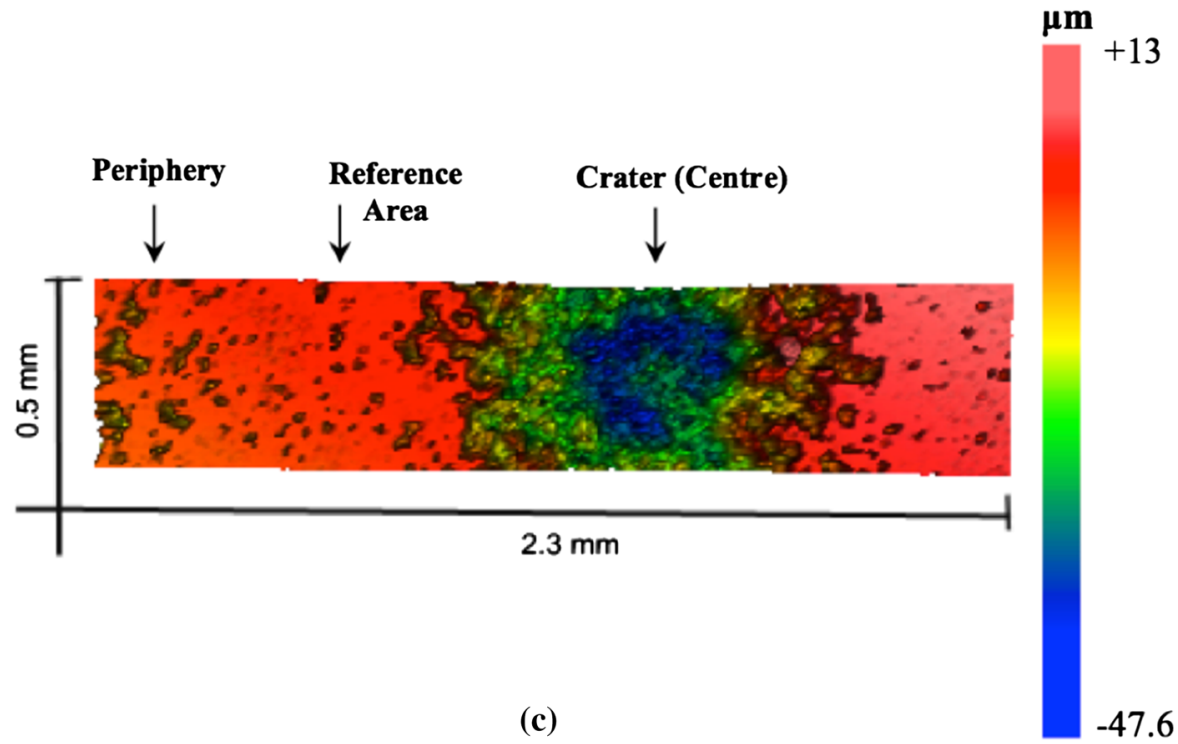

(c)

Fig. 5-Details of a cavitation ring area: $(a)$ image of the high speed acoustic stream which consists of cavitation bubbles, $(b)$ SEM micrograph of the outer annulus and the crater of the ring region, and (c) ZYGO interferometer image of the eroded area.

roughness increased, while the large craters coalesced and developed further to form a much larger crater, thereby leading to significant material removal. The surface layer was completely removed and a very rough surface was formed. The crater became progressively rougher and deeper with time. At the final stage of 8 hours (Figure 6(b)), erosive wear had sufficiently propagated, penetrating the surface, rapidly increasing the depth of the erosive region (Figure 6(c)).

Figures 6(d) and (e) shows the damage that occurred on the AISI 52100 steel surface. Chromium carbides approximately $1 \mu \mathrm{m}$ in size (appeared on the surface of the sample/blistering effect) emerged to the surface due to the consecutive impacts by the cavitation bubbles creating a reinforced substrate contributing to an increase in the strength of the material against cavitation erosion. ${ }^{[31]}$ The stability of the surface was enhanced by the presence of fine, uniformly distributed carbides (Figure 6(d), in-set). However, when the matrix around these carbides became severely eroded, i.e., after 8 hours, carbides were dislodged out of the surface of the sample. After 8 hours of exposure to cavitation, surface was severely eroded forming a deep crater (Figure 6(f)).

Pits and numerous cracks were formed during the early stages of the cavitation erosion on the surface of the AISI 1085 sample, progressing into deep craters in later time steps (Figures 6(g) and (h)). The cracks were initiated at the surface (dash-dot arrow in Figure 6(g)) and spread perpendicular to it, while they tended to join together with the adjacent pits, forming deeper cracks that eventually transformed into small craters (solid arrows in Figure 6(g)). The absence of undulations and the detachment of large chunks (cleavage) of metal with an approximately diameter of $20 \mu \mathrm{m}$ (dashed arrows in Figure $6(\mathrm{~g})$ ) was an indication of surface brittleness. After sufficient exposure, the formation of microcracks was intensified, leading to crack propagation preferentially along the grain boundaries (intergranular fracture) (Figure 6(h), in-set) and brittle failure of the surface (Figure 6(h)). The erosion morphology is highlighted by the interferometric image taken in the core of the crater showing that the AISI 1085 sample had the best erosion resistance against the rate of penetration from the 

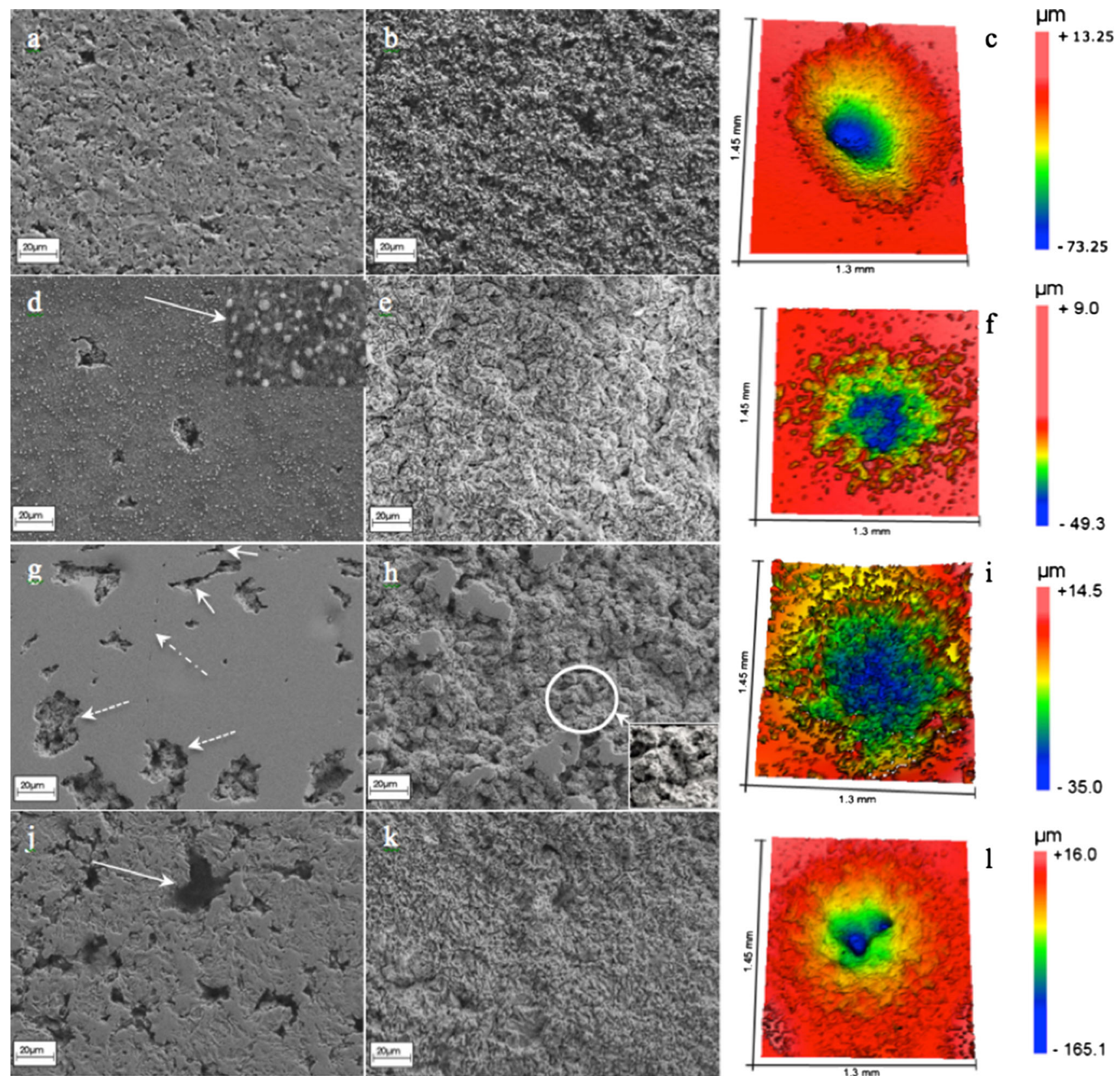

Fig. 6-SEM and ZYGO interferometer micrographs, respectively, showing the morphologies of the steel sample surface after $1(a, d, g, j)$ and 8 $(b, c, e, f, h, i, k, l)$ h exposure to cavitation erosion: $a-c)$ AISI W1, $d-\mathrm{f}$ ) AISI 52100, $g-i$ ) AISI 1085 and $j-l)$ AISI 1020 . (Color legends show the highest and lowest points for each micrograph individually and do not relate to each other. Results are explicitly presented in Table III).

acoustic stream (Figure 6(i)). However, this did not correspond to the highest erosion resistance against cavitation as the size of the crater is significantly larger compared to other steel grades, leading to larger amount of volume loss. A detailed analysis is conducted in Section IV.

As the cavitation erosion progressed on the surface of the AISI 1020, material could not withstand the impacts and started degradation which was notable even after the first hour of treatment (Figure 6(j)). Erosion originated from the cracks, and cleavage of large metal chunks about $50 \mu \mathrm{m}$ (Figure 6(j), white arrow) was also observed. The collapse of bubbles within those cracks in combination with the low hardness of the substrate increased the severity of the cavitation impacts facilitating material removal. The erosion process was further accelerated by a lower resistivity of ferrite to the attack of micro-jets. As a result, it was removed from the surface earlier than the austenite. ${ }^{[44]}$ After 8 hours of testing, the eroded surface morphology was significantly altered, forming a deep valley and very significant loss of material was noticed (Figure 6(1)). The AISI 1020 had the deepest crater among the samples, showing a tendency to cavitation damage while its erosion resistance was very poor in comparison with the other candidate materials. 
The experimental outcomes of the current study (macro-scale) are in agreement with the results obtained earlier ${ }^{[31]}$ for the incubation period of cavitation pits (micro-scale) for the same steel grades, showing that cavitation damage during the incubation stage is in a good agreement with the corresponding cavitation erosion at the final stage.

\section{ANALYSIS AND MECHANISMS}

The cavitation tests were carried out until a clear tendency had been established as explained in Section II-C. The relative resistance of a material to the cavitation erosion can be characterized through measuring the volume loss. The volume loss measured at different times under cavitation erosion is plotted against the treatment time in Figure 7. In general, there was negligible loss of the volume (mass) for all the studied steel grades during the incubation period of cavitation erosion. Volume loss commenced almost immediately after 1 hour exposure to cavitation in all the tests and subsequently increased at a nearly constant rate. It became obvious that AISI 52100 reached the declining period first as the slope curve started to reduce after 7 hours of exposure to cavitation. On the whole, the volume loss in AISI 52100 at the end of the tests was the lowest, approximately 52 pct less as compared with AISI 1020 and 28 pet with AISI W1, indicating the lowest maximum erosion rate among the tested steel grades.

The variation of the MDER parameter with the exposure time is presented in Figure 8. It can be seen that in the first hour, the erosion rate increased very slowly for all the test samples, which is a typical feature of the incubation period. At this stage, due to the accumulated internal stresses across the surface layers, plastic deformation initiated and the material surface started to deform (work hardening). Thus, negligible

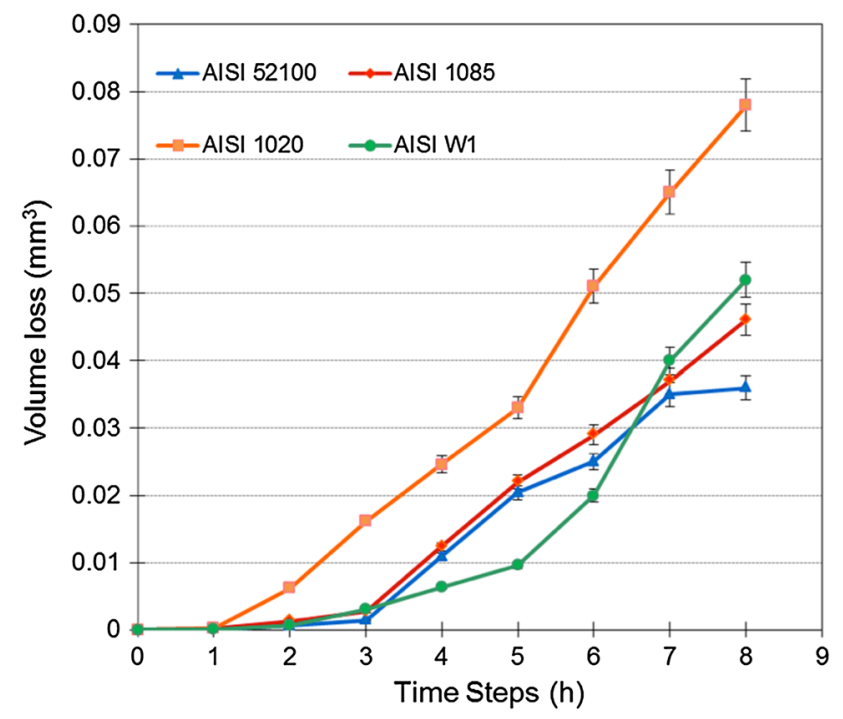

Fig. 7-Cumulative volume loss as a function of time for the examined materials under cavitation erosion. material loss was observed, although fatigue process could already occur during this period. ${ }^{[45]}$ Following this incubation period, the erosion process accelerated. For AISI 1020, the erosion rate started to increase after the first 1 hour of the test and continued until the end of 3 hours where a small attenuation was observed accompanied by a further increment after the end of 5 hours test. There is no evidence of reducing the MDER even after the end of the maximum run test at 8 hours. Thus, the 1 hour of exposure can be considered as the critical point after which AISI 1020 steel significantly decreases its strength and losses its durability. Conversely, for the other tested steel grades the erosion rate gradually increased during the first 3 hours whereupon the acceleration of erosion was observed. This behavior showed that the material was plastically deformed apparently exceeding its fatigue strength limit, which resulted in sudden fast fracture and formation of deep craters. ${ }^{[46]}$ In these stages, evolution of microcracks to macrocracks occurred, and large material removal was observed (see Figure 6). After the end of the acceleration stage, a local equilibrium between the erosive power of the cavitation field and the response of the material was achieved. The erosion process stabilised after 7 hours of exposure, indicating the steady period (change in slope) where the rate of mass loss reached the quasi-constant condition. However, this is the case only for AISI 52100, whereas the other steel grades exhibited an incremental rise in the erosion rate. The endurance of AISI W1 steel after 5 hours was considerably reduced, and the erosion rate steeply increased. Hence, over a prolonged period of exposure to cavitation impacts, the AISI W1 steel plate faced inevitable severe damage. Overall, the erosion rate of the AISI 52100 steel was measured to be the lowest among the samples, while its resistance was calculated to be the highest.

Figure 9 presents the evolution of the roughness profile distribution by means of the $R a$ parameter. Changes in the surface morphology can be clearly observed after the incubation time. The longer the testing time, the higher the number of undulations that were observed across the surface of the steel samples. As the testing time progressed, the undulations widened and deepened. When the steady state was reached, the new roughness might affect the cavity dynamics, and entrapped gas and liquid in the deep craters started cushioning the bubble collapse, consequently restricting further damage. ${ }^{[47]}$

The shapes of roughness profiles were similar with the exception of the AISI W1 steel (Figure 9) where the restriction of extensive surface undulations and damage patterns (Table III) during the early stages of the erosion delayed the progression of roughness. At the beginning of the erosion process, the roughness profile for the AISI 1020 significantly increased, keeping a linear trend during the following hours and reaching a value of more than $20 \mu \mathrm{m}$. In this final stage, erosion could be considered very severe with large craters dominating the damaged surface (Figure 6(1)). The AISI 52100 and AISI 1085 steel samples had a similar roughness profile evolution showing a much smoother growth of their Ra values with time. Within the first 3 
hours, AISI W1 steel exhibited an RA increment of about $4 \mu \mathrm{m}$. Then a quasi-steady period followed between the 3 and 5 hours steps. The lowest erosion rate among the tested steel grades during that stage was

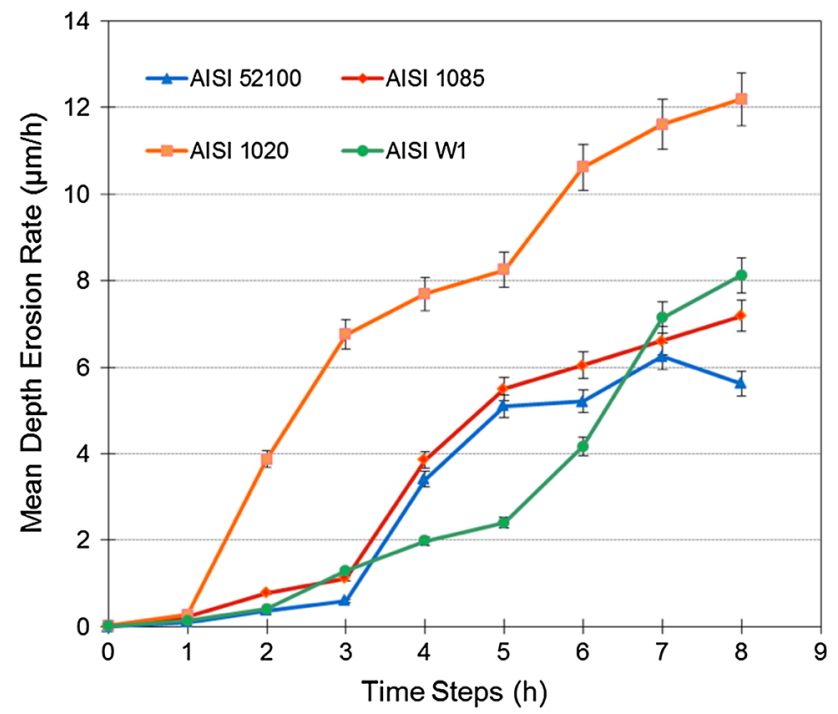

Fig. 8-Variation of the MDER parameter with the exposure time for different steel materials.

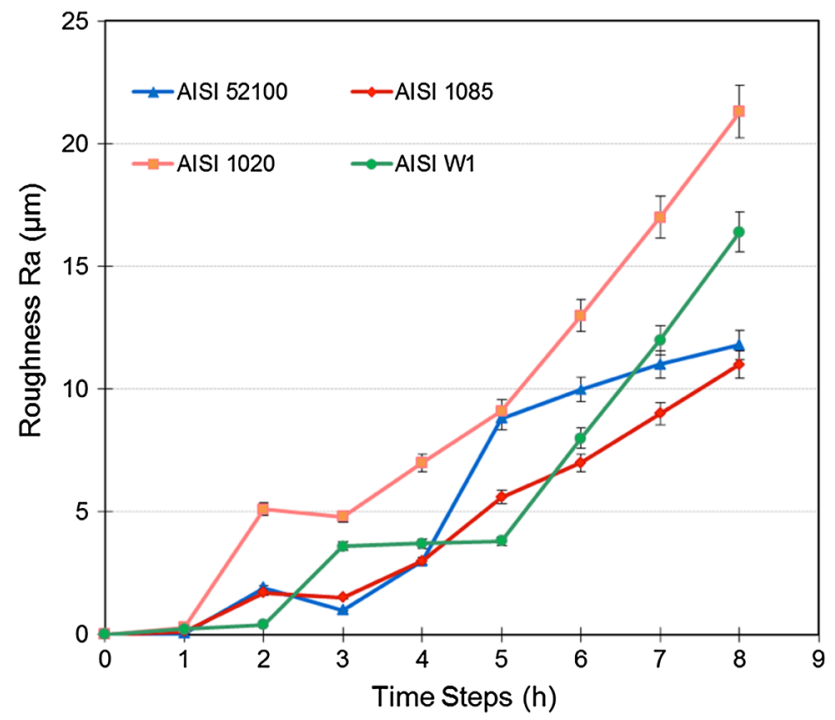

Fig. 9-Results of the surface roughness measurements showing the variation of $R a$ with the exposure time of the different steel grades. achieved by AISI W1 as can be seen from Figure 8. Then, roughness seemed to drastically increase after 5 hours reaching a $R a$ value of more than $15 \mu \mathrm{m}$.

Figure 10 presents the data from Figures 8 and 9 normalized to the maximum value. This was done by dividing each of the measured values with the maximum measured value among all the variables. For example, the volume loss used in Eq. [1] to calculate the MDER in each case was divided by the maximum volume loss so a unit-based normalization to the maximum value was achieved. In this way, all the experimental variables from these sets of experiments were related giving meaningful generalized results.

The normalized data (Figure 10) for both of the quantities illustrates a very good fit among the different steel samples that can be expressed with Eq. [6], where $a$ is 0.96 and $n$ is 1.25 . Note that specific tests cover different ranges of the erosion curve, but all test data fall on one common erosion curve with a satisfactory cross correlation factor $R^{2}=0.93$. These results are in agreement with ${ }^{[48,49]}$ showing that the roughness of the eroded surface is related with the mass detachment process under cavitation erosion conditions.

$$
V / V_{\max }=a\left(R_{a / R_{\max }}\right)^{n}
$$

The erosion damage experienced by the different steel grades was also characterized by measuring the maximum height difference between the centre of the eroded surface and the original surface due to material loss and the results are summarized in Table III.

As described in the experimental procedure, the evaluation of the individual damage periods was performed by considering the $I_{D}$ parameter of Eq. [5]. Specifically, the maximum depth of the damaged area showed a rapid increment during the first 2 hours as cavitation moved from the incubation to the acceleration stage. The increment of the exposure time lead to an increasing rate of around 30 to 40 pct until 3 hours except in the case of AISI W1 where a rapid increment of the crater depth was observed at around 50 pct. During the period between 3 and 5 hours of exposure, the crater growth rate is significantly increased within the range of 50 to $65 \mathrm{pct}$ for all the steel samples except for the AISI W1 steel (30 pct). In this time step, failure of the matrix to withstand more cavitation impacts lead the exposed surface of AISI W1 steel to rapid degradation. Furthermore, the transition between the acceleration stage and the maximum erosion rate stage was also

Table III. Maximum Penetration Depth of the Eroded Areas at Different Time Steps

\begin{tabular}{|c|c|c|c|c|c|c|c|c|}
\hline \multicolumn{9}{|c|}{ Maximum Depth $(\mu \mathrm{m})$} \\
\hline Time Steps (h) & 1 & 2 & 3 & 4 & 5 & 6 & 7 & 8 \\
\hline Formation Pattern & Pits & Crater & Crater & Crater & Crater & Crater & Crater & Crater \\
\hline AISI 52100 & $<1$ & $9 \pm 0.5$ & $13 \pm 0.5$ & $28 \pm 1$ & $40.5 \pm 1$ & $44 \pm 1$ & $53 \pm 1$ & $54 \pm 1.5$ \\
\hline AISI 1085 & $<1$ & $11 \pm 0.5$ & $16 \pm 0.5$ & $20 \pm 0.5$ & $23 \pm 1$ & $29 \pm 0.5$ & $37 \pm 1$ & $46 \pm 2$ \\
\hline AISI 1020 & $\leq 2$ & $22 \pm 1$ & $33 \pm 1.5$ & $50 \pm 1.5$ & $62 . \overline{3} \pm 2$ & $95 \pm 1.5$ & $128 \pm 2$ & $170 \pm 5$ \\
\hline AISI W1 & $<1$ & $8 \pm 0.5$ & $16 \pm 0.5$ & $19 \pm 0.5$ & $22 \pm 1.5$ & $41 \pm 2$ & $62 \pm 1.5$ & $78 \pm 2$ \\
\hline
\end{tabular}


taking place. In the final stage, from 5 to 8 hours, small increment of the depth of the craters was observed for the AISI 52100 with a crater growth rate dropping to 25 pct, implying the establishment of a steady state damage period (terminal stage). On the contrary, the AISI 1085, AISI 1020, and AISI W1 steels still exhibited higher crater growth rates around 50, 65, and 70 pct, respectively.

Figure 11 presents data normalized to the maximum value of the accumulated strain energy with the volume loss according to Eqs. [3] and [4] with the data taken from Table II and Figure 8 in the case of brittle behavior (Figure 11(a)) and in the case of elastoplastic behavior (Figure 11(b)). The AISI 52100 steel exhibits

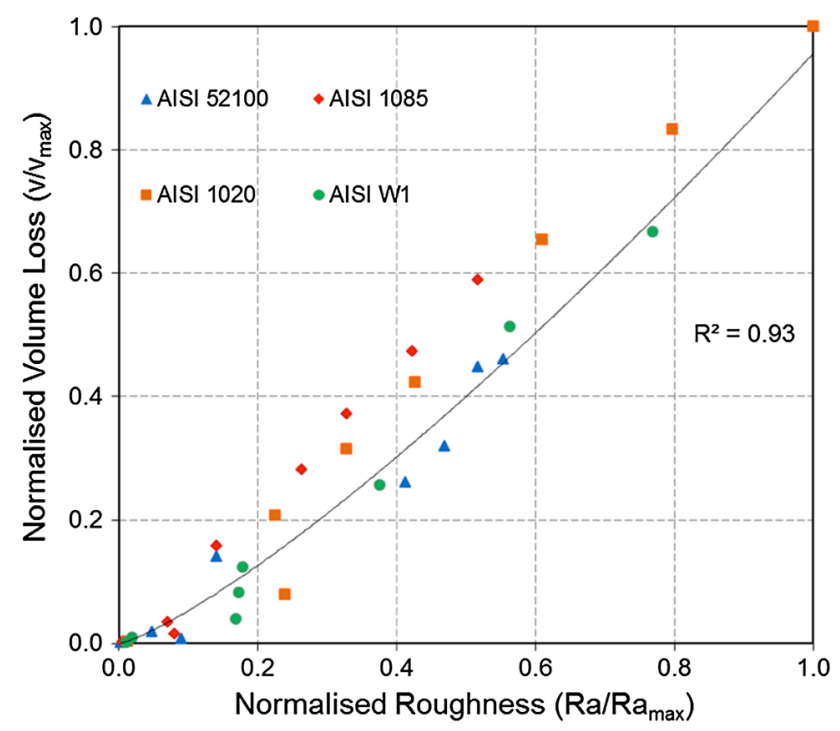

Fig. 10 - Curves of the normalized volume loss vs normalized $R a$ roughness parameter. the best cavitation erosion performance among the tested steel samples, i.e., for the same time exposure to cavitation damage (i.e., 8 hours) much more accumulated strain energy is required in order to remove the same amount of material.

Specifically, it can be seen that in the case where only the elastic strain energy is considered (Figure 11(a)). AISI 52100 has superior behavior among the other materials closely followed by the AISI W1. Although when the accumulated total strain energy is considered (Figure 11(b)), it is apparent that the AISI 52100 exhibits the best performance (being below the trend curve) as for the same exposure time to cavitation requires much more strain energy in order to reach similar volume loss.

In the case where the elastic energy is plotted against the volume loss (Figure 11(a)), there is poor correlation as data points are scattered. When the total accumulated energy was considered (see Figure 11(b)), implying a more elastoplastic behavior of the material, the volume loss shows an excellent correlation with total strain energy $\sigma^{*}$ which can be expressed with Eq. [7] where $a$ is 0.9 and $n$ is 0.99 .

$$
V / V_{\max }=a\left(\sigma^{*} / \sigma_{\max }^{*}\right)^{n}
$$

Therefore, our results demonstrate that cavitation erosion is strongly $\left(R^{2}=0.98\right)$ correlated with the elastoplastic behavior (toughness and plasticity) of the tested materials, which can be applied to a wider range of materials.

Additionally, results clearly showed that $\mathrm{Cr}$ containing alloy steel grades exhibited a much more prominent resistance against cavitation damage as compared to the rest of the studied steel grades. For example, in the case of AISI 52100 accumulated strain energy needed to be almost twice as much as for AISI 1085 or AISI W1 in

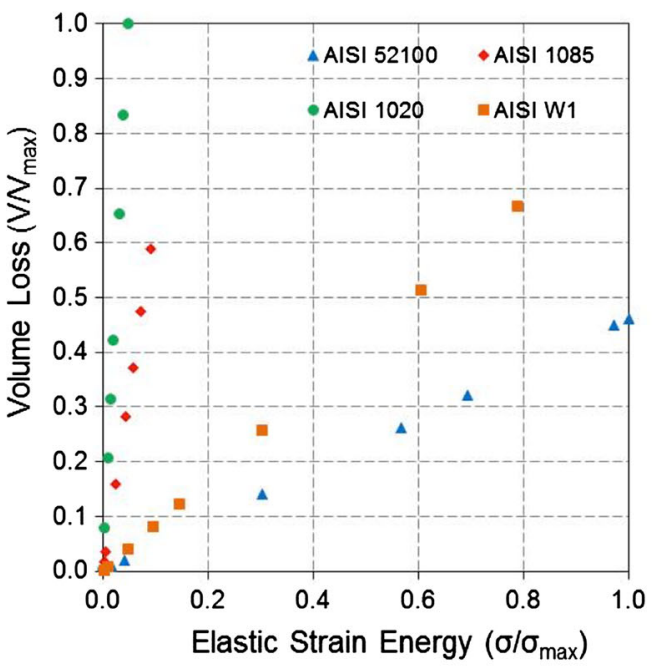

(a)

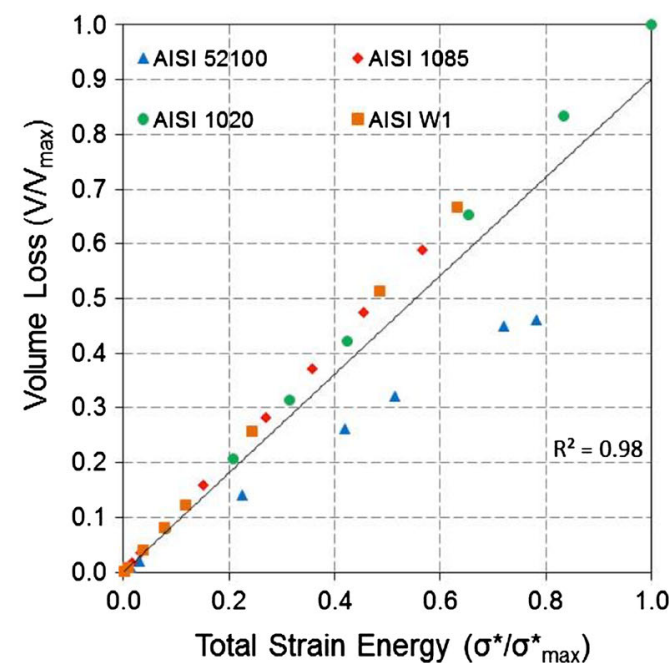

(b)

Fig. 11- General behavior of the tested steel grades is shown by the normalization of $(a)$ elastic accumulated strain energy $E_{\mathrm{el}}$ and $(b)$ total accumulated strain energy $E_{\text {tot }}$ with the variation of the volume loss. All curves start from the origin, but for the same physical time, the tested materials reached different erosion stages (each point represents an hour time step up to $8 \mathrm{~h}$ ). 
Table IV. Variation of Hardness Vickers with the Cavitation Exposure Time

\begin{tabular}{|c|c|c|c|c|}
\hline Exposure Time (h) & AISI 52100 & AISI 1085 & AISI 1020 & AISI W1 \\
\hline 1 & $891 \pm 4$ & $805 \pm 2$ & $305 \pm 3$ & $571 \pm 3$ \\
\hline 2 & $805 \pm 1$ & $686 \pm 1$ & $289 \pm 6$ & $552 \pm 3$ \\
\hline 3 & $759 \pm 7$ & $661 \pm 3$ & $269 \pm 5$ & $474 \pm 6$ \\
\hline 4 & $722 \pm 5$ & $616 \pm 2$ & $232 \pm 4$ & $467 \pm 2$ \\
\hline 5 & $687 \pm 8$ & $571 \pm 1$ & $195 \pm 4$ & $460 \pm 2$ \\
\hline 6 & $651 \pm 2$ & $526 \pm 3$ & $187 \pm 2$ & $452 \pm 2$ \\
\hline 7 & $645 \pm 1$ & $499 \pm 3$ & $180 \pm 2$ & $434 \pm 2$ \\
\hline 8 & $636 \pm 2$ & $475 \pm 8$ & $174 \pm 3$ & $427 \pm 3$ \\
\hline
\end{tabular}

order to remove similar amount of mass (see data points in the region between 0.4 and 0.5 in the $y$ axis of Figure 11(b)).

For many steel grades, the microstructural features and the dislocation structure affect their properties, e.g., hardness and, consequently, their cavitation erosion resistance. ${ }^{[50]}$ Many research studies have agreed that cavitation erosion damage strongly depends on the hardness alterations across the sample surface.$^{[34,51]}$ As the material is exposed to great impulsive pressures by the collapse of cavitation bubbles, the successive hydrodynamic impacts lead to a progressive work hardening resulting in the strain accumulation in the vicinity of the impact zone. Also, the formation of new dislocations causes dislocation blockade and motion restriction while the dislocation density increases especially along the grain boundaries and along the eroded surface, resulting in a higher local hardness. ${ }^{[52]}$ On the other hand, the heat generated by the cavitation process, the repeated impact pressures and the interactions between the dislocations and the grain structure may cause softening, i.e., hardness drop. ${ }^{[21]}$

In Table IV and Figure 12, the cavitation erosion resistance (CER) was evaluated in terms of hardness. It can be noticed that the drop of hardness after the end of cavitation incubation stage was very rapid for every material (compare initial hardness in Table II and measurements in Table IV). For the AISI 52100 and W1 steel grades, work hardening occurred during the first hour of cavitation erosion. Hardness increased by 2.5 and 5.5 pct for AISI 52100 and $\mathrm{W} 1$, respectively. There is a possibility (based on previous observations in References 31) that the changes in microstructure of the both steel grades resulted in the increased surface hardness due to martensite deformation within the martensitic laths, and then in the hardness decreased as a result of fatigue crack initiation and propagation, local fracture, and subsequent crater formation. In contrast, for the remaining two steel grades the hardness dropped to about $8.5 \mathrm{pct}$ for the AISI 1085 and $15 \mathrm{pct}$ for the AISI 1020 during the same period of time. AISI 1020 with the lowest carbon percentage was incapable of resisting severe impacts from the implosion of the cavitation bubbles as the material lost stability and experienced accelerated damage. Hardness reduction of AISI 52100 and AISI W1 steel grades during the testing period of 8 hours reached 26 and 21 pct, respectively, while for AISI 1085 and 1020 steel grades it reached 46 and $51 \mathrm{pct}$, correspondingly. These data clearly highlight

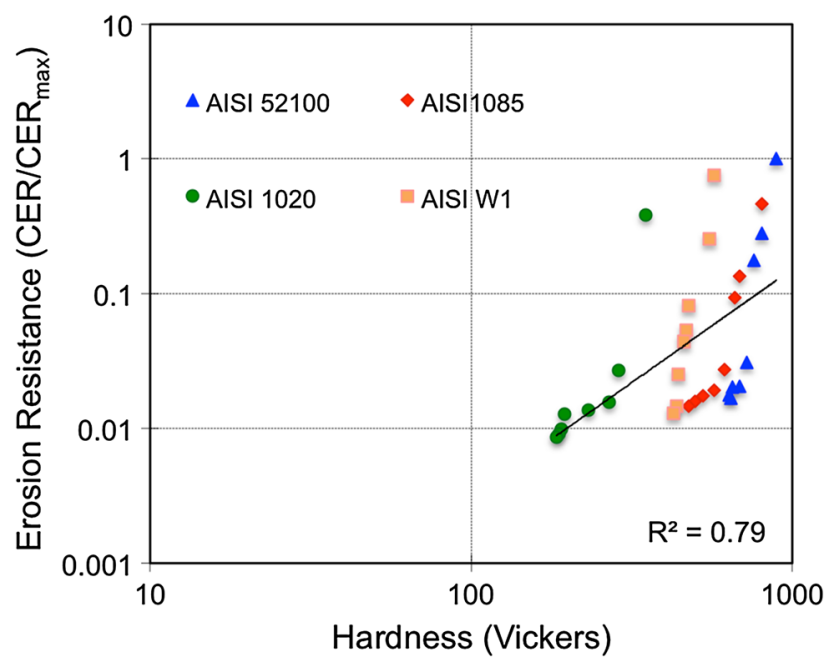

Fig. 12 - Correlation of hardness to the cavitation erosion resistance for the studied steel grades (normalization curves).

the counterbalancing effect of the work hardening component in the evaluation of durability of the material against cavitation and the attenuation of the micro-durability of the material as also shown by Krella. ${ }^{[53]}$ The overall behavior of the erosion resistance can be expressed as a function of the hardness $H$ as per Eq. [8] where $a$ is 0.9 and $n$ is $7.8\left(R^{2}=0.79\right)$ (Figure 12):

$$
\mathrm{CER} / \mathrm{CER}_{\max }=a(H)^{n}
$$

Hence, the erosion resistance of an arbitrary material, with mechanical and physical properties in the range of the tested steel grades, increases, following a power relationship with the hardness of the material. It is evident that the CER of steel grades is dependent on their hardness, although this does not necessarily mean, as shown in this study, that the material with a higher hardness will exhibit a better performance against cavitation erosion as other structural and mechanical factors play significant role as well.

\section{SUMMARY AND CONCLUSIONS}

In the current study, a comparison between the steel material AISI W1 currently used in a scroll expander 
system and three commercially available steel grades AISI 52100, AISI 1085 and AISI 1020, which are typically utilized for fluid-machinery manufacturing, is conducted. A thorough post-test analysis was performed based on their cavitation resistance. The comparison of the erosion rates from the accelerated erosion tests showed that the erosion response of the studied steel grades is linked to the characteristics of the material such as tensile properties, accumulated strain energy and hardness. Consequently, the tested materials were ranked based on their cavitation resistance, with AISI 52100 exhibiting the best performance.

The main conclusions can be listed as follows:

1. The evaluation of the SEM and optical interferometric images showed that erosion development is characterized by the formation of erosion pits and deep craters. The resulting material damage at the earlier stage of cavitation erosion differs among the tested steel samples; however, at their final stage a similar brittle fracture is shown.

2. The superior performance of the AISI 52100 steel against the other steels is shown. AISI 52100 exhibits the best cavitation erosion resistance followed by the AISI 1085. The actual steel material of the scroll expander AISI W1 and the AISI 1020 showed a very poor resistance against cavitation at longer time steps. The main advantage of AISI 52100 is that at longer exposure times the erosion damage growth is controlled, preventing any steep increment of the erosion rate.

3. The present study indicates that roughness measurement may constitute a practical method for monitoring damage within industrial fluid machinery equipment. A generalized approach of the roughness profile in correlation to the volume removed is given by the power relationship $V / V_{\max }=a\left(R_{a / R_{\max }}\right)^{n}$ with a satisfactory correlation coefficient of 0.93 .

4. The accumulated strain energy plays a significant role in resisting cavitation erosion over the entire testing duration. It was shown that the $\mathrm{Cr}$ containing steel grade exhibited a much more prominent resistance against cavitation damage as compared to the rest of the studied alloy grades. Overall, AISI 52100 possessed the highest cavitation resistance as it has the capacity to absorb more cavitation energy. The normalized total (elastic + plastic) strain energy in correlation to the normalized volume loss is given by the power relationship $V / V_{\max }=a\left(\sigma^{*} / \sigma_{\max }^{*}\right)^{n}$ with a correlation coefficient of 0.98 .

5. The change in hardness is generally closely correlated with the erosion resistance of the steel samples. However, in this study where similar hardness range materials (i.e., AISI 1085 and AISI 52100) were tested, it was clearly shown that a steel grade which has an initial high hardness similar to AISI 1085 is not necessarily superior against cavitation erosion, as the work hardening component during the early stages of cavitation also has to be considered. The normalized erosion resistance can be expressed on the basis of dimensionless parameter related to hardness of the material as $\mathrm{CER} / \mathrm{CER}_{\max }=a(H)^{n}$ with a reasonable correlation coefficient at 0.79 .

\section{OPEN ACCESS}

This article is distributed under the terms of the Creative Commons Attribution 4.0 International License (http://creativecommons.org/licenses/by/4.0/), which permits unrestricted use, distribution, and reproduction in any medium, provided you give appropriate credit to the original author(s) and the source, provide a link to the Creative Commons license, and indicate if changes were made.

\section{REFERENCES}

1. I. Tzanakis, M. Hadfield, A. Georgoulas, and N. Kotsovinos: WIT Trans. Eng. Sci., 2010, vol. 6, pp. 261-72.

2. I. Tzanakis, A. Georgoulas, M. Hadfield, and N. Kotsovinos: Int. J. Comput. Methods Exp. Meas. (CMEM), 2010, vol. 2, pp. 168-83.

3. I. Tzanakis, D. Eskin, A. Georgoulas, and D. Fytanidis: Ultrason. Sonochem., 2014, vol. 21, pp. 886-78.

4. I. Tzanakis, A. Georgoulas, D. Fytanidis, M. Hadfield, N. Kotsovinos: Proceedings World Tribology Congress, Turin, Italy, 2013, vol 1, pp. 947-51.

5. C.M. Hansson, I.L.H. Hansson ASM Handbook, Friction, Lubrication, and Wear Technology (ASM International), vol. 181992, pp. $214-70$

6. A. Harkin, A. Nadim, and T.J. Kaper: Phys. Fluids, 1999, vol. 11, pp. 274-87.

7. E.A. Brujan, T. Ikeda, and Y. Matsumoto: Soft Matter, 2012, vol. 21 , pp. $5777-83$.

8. I. Tzanakis, A. Georgoulas, M. Hadfield, and N. Kotsovinos: WIT Trans. Eng. Sci., 2012, vol. 76, pp. 129-37.

9. Howell, P. D.: Cambridge University Press, Cambridge, UK, 2001, pp. 32-56.

10. I. Tzanakis, M. Hadfield, and Z. Khan: WIT Trans. Eng. Sci., 2009, vol. 62, pp. 229-40.

11. J.P. Franc: J. Fluids Eng., 2009, vol. 131, pp. 0213031-02130314.

12. A. Karimi and J.L. Martin: Int. Met. Rev., 1986, vol. 31 (1), pp. 1-26.

13. M.C. Park, K.N. Kim, G.S. Shin, and S.J. Kim: Wear, 2012, vols. $274-275$, pp. $28-33$.

14. R. Garcia and F.G. Hammitt: Trans. ASME, 1967, vol. 89, pp. 753-63.

15. S. Hattori and T. Kitagawa: Wear, 2010, vol. 269, pp. 443-48.

16. J.D. Escobar, E. Velasquez, T.F.A. Santos, A.J. Ramirez, and D. Lopez: Wear, 2013, vol. 297, pp. 998-1005.

17. C. Wenge, G.U. Cheonquing, Z. Kang, and S. Fusan: J. Mater. Sci., 2006, vol. 41, pp. 2151-53.

18. I. Tzanakis, M. Hadfield, and I. Henshaw: Exp. Therm. Fluid Sci., 2011 , vol. 35 , pp. $1544-54$

19. R. Zhao, R. Xu, Z. Shen, J. Lu, and X. Ni: Opt. Laser Technol., 2007, vol. 39, pp. 968-72.

20. Y. Meged, C.H. Venner, and W.E. ten Napel: Wear, 1995, vol. 186 , pp. $443-53$.

21. A. Krella: Wear, 2005, vol. 258, pp. 1723-31.

22. M.D. Kass, J.H. Whealton, N.E. Clapp, Jr, J.R. DiStefano, J.H. DeVan, J.R. Haines, M.A. Akerman, and T.A. Gabriel: Tribol. Lett., 1998, vol. 5, pp. 231-34.

23. I. Tzanakis, M. Hodnett, B. Lebon, D.G. Eskin, and K Pericleous: Sens. Actuators, 2016, vol. 240, pp. 57-69.

24. S. Hattori, T. Ogiso, Y. Minami, and I Yamada: Wear, 2008, vol. 265, pp. 1619-25. 
25. S. Hattori and R. Ishikura: Wear, 2010, vol. 268, pp. 109-16.

26. C. Haosheng, L. Jiang, C. Darong, and W. Jiadao: Wear, 2008, vol. 265, pp. 692-98.

27. M. Dojcinovic, O. Eric, D. Rajnovic, L. Sidjanin, and S. Balos: Mater. Charact., 2013, vol. 82, pp. 66-72.

28. J. Steller: Wear, 1999, vols. 233-235, pp. 51-54.

29. K.Y. Chiu, F.T. Cheng, and H.C. Man: Ultrasonics, 2005, vol. 43, pp. 713-16.

30. C.T. Kwok, F.T. Cheng, and H.C. Man: Surf. Coat. Technol., 2006, vol. 200, pp. 3544-52.

31. I. Tzanakis, N. Garland, and M. Hadfield: Tribol. Int., 2011, vol. 44, pp. 1668-78.

32. K. Kim, H. Chahine, G. Franc, J.P. Karimi: Advanced Experimental and Numerical Techniques for Cavitation Erosion Prediction. Springer, vol 106, 2014.

33. F. Dong, X. Li, L. Zhang, L. Ma, and R. Li: Ultrason. Sonochem., 2016, vol. 31, pp. 150-56.

34. F.G. Hammitt: Cavitation and Multiphase Flow Phenomena, McGraw-Hill, New York, 1980.

35. H. Date and M. Futakawa: Int. J. Impact Eng., 2005, vol. 32, pp. 118-29.

36. Thiruvengadam S. Waring: Hydronautics Inc Laurel MD, Technical Report (1964) HYDRO-TR-233-5.

37. I. Tzanakis PhD Thesis, Bournemouth University, 2010 (http://eprints.bournemouth.ac.uk/16060/2.

38. I. Tzanakis, M. Hadfield, I. Hensaw, N. Garland, and Z. Khan: Tribol. Trans., 2011, vol. 54, pp. 505-13.
39. I. Tzanakis, M. Conte, M. Hadfield, and T.A. Stolarski: Wear, 2013, vol. 303, pp. 154-68.

40. M. Dular, O.C. Delgosha, and M. Petkovšek: Ultrason. Sonochem., 2013, vol. 20, pp. 1113-20.

41. P.D. Howell: Mathematical Modelling: Case Studies from Industry. Mathematics for Science and Engineering, Cambridge University Press, Cambridge, UK, 2001, pp. 32-56.

42. G. García-Atance Fatjó, A. Torres Pérez, M. Hadfield: Ultrason. Sonochem., 2010, vol 17, pp. 73-79.

43. A. Moussatov, C. Granger, and B. Dubus: Ultrason. Sonochem., 2003, vol. 10, pp. 191-95.

44. A. Karabenciov, A.D. Jurchela, I. Bordeașu, M. Popoviciu, N. Birău, and A. Lustyan: IOP Conf. Ser., 2010, vol. 12, p. 012036.

45. G.L. García, V. López-Ríos, A. Espinosa, J. Abenojar, F. Velasco, and A. Toro: Wear, 2014, vol. 316, pp. 124-32.

46. J. Steller, A. Krella, J. Koronowicz, and W. Janicki: Wear, 2005, vol. 258, pp. 604-13.

47. J. Choi, A. Jayaprakash, and G.L. Chahine: Wear, 2012, vols. 278-279, pp. 53-61.

48. M. Pohl and J. Stella: Wear, 2002, vol. 252 (5-6), pp. 501-11.

49. J.D. Escobar, E. Velasquez, T. Santos, A. Ramirez, and D. Lopez: Wear, 2013, vol. 297, pp. 998-1005.

50. A. Krella and A. Czyzniewski: Wear, 2007, vol. 263, pp. 395-401.

51. S. Hattori and T. Kitagawa: Wear, 2010, vol. 269, pp. 443-48.

52. A. Krella, A. Zielinski: Proceedings of the Ninth International Scientific Conference AMME, 2000.

53. A. Krella: Surf. Coat. Technol., 2009, vol. 204, pp. 263-70. 